\section{National forest inventories: contributions to forest biodiversity assessments (2010)}

\author{
Motta R \\ National Forest Inventories are the most complete source of information on \\ forests, and are also a potential important tool for assess and monitoring status \\ and trends in forest biodiversity, Nevertheless, according to Chirici et al., the \\ current inventories have some limitations and should be improved mainly to in- \\ crease the harmonisation of the field protocols and to enhance the collection \\ of non-tree forest information that are important for the biodiversity assess- \\ ment.
}

Keywords: Forest inventory, Biodiversity, Sustainable management, Natura 2000

Gherardo Chirici, Susanne Winter, Ronald E. McRoberts (eds.). "National forest inventories: contributions to forest biodiversity assessments". Series "Managing Forest Ecosystems", vol. 20, Springer Ed., Dordrecht, The Netherlands, pp. 206.

This edited book has 26 contributors from 10 different countries and it is the result of the activities of Working Group 3 (WG3): "Contributions of field data acquired in NFI for forest biodiversity assessments" led by Gherardo Chirici and Susanne Winter of COST Action E43 "Harmonisation of National Inventories in Europe: Techniques for Common Reporting" (chaired by Prof. Erkki Tomppo). The main purpose of the book is to provide a comprehensive and informative overview of forest biodiversity and to analyse the potential role of National Forest Inventories in providing robust forest biodiversity indicators.

The issue of the conservation of the biodiversity has been taken into consideration in most Forest management plans for long time but it is only since 1992, the year in which a large number of countries signed the Convention on Biological Diversity in Rio de Janeiro, that the issues to monitor, protect and enhance biodiversity have been included

$\square$ Dip. AgroSelviTer, University of Turin (Italy)

@ Renzo Motta (renzo.motta@unito.it)

Received: May 11, 2011 - Accepted: May 17, 2011

Citation: Motta R, 2011. National forest inventories: contributions to forest biodiversity assessments (2010). iForest 4: 250-251 [online 2011-11-03] URL: http://www.sisef.it/iforest/show.php? id $=577$ officially in most national and international forestry policies. Besides in the last decades International Conventions and the increasing awareness of the stakeholders and policy and decision makers have pointed out the need for robust and statistically sound, updated and long-term information on non-productive forest functions and on the maintenance of biodiversity.

The most extensive and comprehensive data on the status of the forests at the national and/or regional levels are yet provided by the Forest inventories that have been established in many countries.

Methods of forest inventory have principally been developed for estimating the standing volume of wood in forests and for recurrent measurements to indicate changes in stand structure and growth with time. Traditionally, such inventories focus on assessing timber yield, and therefore do not generally incorporate measures of other ecosystem components. Besides due to different historical backgrounds and varying environmental conditions National forest inventories use different basic definitions and methods, which lead to inconsistencies and lack of harmonize global forest information international organisations (e.g., FAO) have been developing common key definitions. Nevertheless these definitions have not been harmonized and consistently applied.

In the same time many research projects have developed efficient, optimized methods for monitoring forest biodiversity at a variety of spatial and temporal scales. Nevertheless, a straightforward approach for monitoring forest biodiversity is still lacking.

The book is divided into 6 chapters.

In Chapter 1 (The Need for Harmonized Estimates of Forest Biodiversity Indicators) the authors analyse the problems related to the harmonization that typically consists of comparability for international reporting. To two components: development of common international reference definitions and development bridging techniques that facilitate estimation according to reference definitions using data collected according to national definitions.

Chapter 2 (Essential Features of Forest Biodiversity for Assessment Purposes) describes how Working Group 3 (WG3) of COST Action E43 selected 41 candidate biodiversity variables based on current ecological knowledge and the process that lead to the selection of 17 biodiversity variables that were then grouped into seven essential biodiversity features: forest categories, forest age, forest structure, deadwood, regeneration, ground vegetation and naturalness.

Chapter 3 (Prospects for Harmonized Biodiversity Assessments Using National Forest Inventory Data) evaluated the prospects for harmonized estimates of biodiversity indicators based on selected variables. The evaluations produced positive harmonization possibilities for forest categories and the tree height and diameter variables associated with forest structure. For other variables, possibilities were constrained by lack of common reference definition, assessment methods, measurement thresholds and other factors. Thus, efforts at harmonization for these essential features were constrained to a few variables or a few countries with similar NFI features.

In Chapter 4 (The Common NFI Database) bridging techniques for the harmonized estimation of forest biodiversity indicators for each of the selected essential features were tested and a common database was constructed and populated with raw NFI data provided by some of the COST Action E43 participating countries, while Chapter 5 (Harmonization Tests) reports the results of testing the proposed procedures for harmonizing estimates of indicators for six of the seven essential features of forest biodiversity. Finally, Chapter 6 provides summary and conclusions. The main conclusions are that National Forest Inventories already are the most complete source of information on forests, both in terms of number of sampling units and in terms of the information acquired in the field and are also a potential important source also for assess and monitoring trends in forest biodiversity, Nevertheless the inventories have some limitations and should be improved mainly to increase the harmonisation of the field protocols and to enhance the collection of non-tree forest information that are important for the biodiversity assessment.

The book is clearly written even if it is rich in definitions, references to previous projects and acronyms that require a lot of attention and makes the reading challenging for a nonspecialist. The monitoring of forest and natu- 
ral resources are becoming more and more important for the application of a sustainable management, the biodiversity, the carbon budget and so on. It is crucial to develop tools that are harmonized in definition adopted and methods and take into account all the requirements. This book clearly describes the state of art of this field of applied research and makes some proposals in order to make the most of what has already be done and to address the further developments. Both readers that are interested in Forest In- ventories (at the national or at the local scale) or readers that are interested in biodiversity assessment or monitoring would benefit from reading this book, which helps to narrow the gap between forestry and natural resource managements. 